\title{
EFICACIA, SEGURIDAD Y EFICIENCIA DE LAS NUEVAS TECNOLOGÍAS EN CIRUGÍA TIROIDEA*
}

\author{
Drs. Benito Flores P. ${ }^{1}$, Joana Miguel P. ${ }^{1}$, Víctor Soria A. ${ }^{2}$, \\ Alfredo Moreno E. ${ }^{1}$, Andrés Carrillo A. ${ }^{3}$, José Luis Aguayo A. ${ }^{2}$
}

\author{
Servicio de Cirugía General. Unidad de Cirugía Endocrina. \\ Servicio de Cirugía General y Digestiva. \\ 3 Servicio de Medicina Intensiva. Coordinador de Investigación. \\ Hospital Universitario Morales Meseguer, Murcia, España.
}

\begin{abstract}
\section{Effectiveness, safety and efficiency of new technologies in thyroid surgery}

Introduction: Surgery of the thyroid gland is the most performed procedure by the endocrine surgeon. In the last years, new techniques have been incorporated in this procedure. Our objective is to analyse the impact of these techniques in our department. Methods: We performed a retrospective and comparative study among three samples of patients submitted for total thyroidectomy. Group I: Sample of 96 patients operated between 2004 and 2007 performing total thyroidectomy. In this period we didn`t use ultrasonic scalpel (US) nor intraoperative neuromonitoring (INM). Group II: Sample of 108 patients operated between 2008 and 2010. In this group we used US for hemostasis. Group III: Sample of 82 patients operated between 2011 and 2012. In this group we used both US and INM. The groups are compared: complications, postoperative stay, surgical time and costs per patient. Results: We analysed the following complications: haemorrhage, postoperative hipocalcemia and recurrent nerve palsy. We didn't find significant differences among the samples of patients but there were a less percentage of recurrent palsy in the third group (4.9\% vs 12.5 and $11.1 \%)$. We found significant differences when stay, surgical time and costs were analysed. Conclusion: The use of new techniques in thyroid surgery has supposed an improvement not only in the clinic outcomes but in the surgical time.
\end{abstract}

Key words: Thyroid surgery, ultrasonic scalpel, intraoperative neuromonitoring, costs.

\section{Resumen}

Objetivo: La cirugía de la glándula tiroides representa el procedimiento más frecuente que realiza el cirujano endocrino. En los últimos años se han ido incorporando nuevas técnicas aplicadas a este procedimiento. El objetivo de nuestro trabajo es analizar el impacto que dichas técnicas han tenido en nuestro servicio. Material y Métodos: Estudio retrospectivo y comparativo entre tres muestras de pacientes a los

*Recibido el 13 de enero de 2014 y aceptado para publicación el 19 de febrero de 2014.

Los autores no refieren conflictos de interés.

Correspondencia: Dr. Benito Flores P.

florespastorbenito2@gmail.com 
que se realizó tiroidectomía total. Grupo I: Muestra de 96 pacientes intervenidos entre 2004 y 2007 a los que se realizó tiroidectomía total. En este período no se utilizó bisturí ultrasónico (BU) ni neuromonitorización intraoperatoria (NMI). Grupo II: Muestra de 108 pacientes intervenidos entre 2008 y 2010. En este grupo se realizó hemostasia con BU. Grupo III: Muestra de 82 pacientes intervenidos entre 2011 y 2012. Se utilizó tanto el BU como la NMI. Se comparan los grupos en cuanto a: complicaciones, estancia postoperatoria, tiempo quirúrgico y coste por paciente. Resultados: Las complicaciones analizadas fueron: hemorragia, hipocalcemia postoperatoria y parálisis del nervio laríngeo recurrente. No encontramos diferencias significativas entre los grupos aunque sí hay una menor tasa de parálisis recurrenciales en el tercer período (4,9\% vs 12,5 y $11,1 \%)$. Hemos obtenido diferencias significativas en estancia, tiempo quirúrgico y coste por paciente. Conclusión: La inclusión de las nuevas técnicas en cirugía tiroidea ha supuesto una mejora en los resultados clínicos así como un ahorro de tiempo de quirófano.

Palabras clave: Cirugía tiroidea, bisturí ultrasónico, neuromonitorización intraoperatoria, costes.

\section{Introducción}

La práctica de la tiroidectomía ha evolucionado de forma constante desde sus inicios como proceder científico a mediados del siglo XIX ${ }^{1}$. Las altas tasas de mortalidad, directa e indirecta, atribuibles a esta técnica en un principio, han quedado reducidas hoy en día a cifras insignificantes. Otras complicaciones, sin embargo, aunque infrecuentes, siguen estando presentes en la mente tanto de pacientes como de los cirujanos endocrinos: hemorragia postoperatoria, hipoparatiroidismo y parálisis recurrencial. Factores predictivos de estas complicaciones no son fáciles de identificar. Es por ello que una cuidadosa técnica quirúrgica, adecuada hemostasia e identificación sistemática de estructuras han sido los pilares en la prevención de complicaciones ${ }^{1-3}$.

Desde hace unos años, nuevas tecnologías han ido surgiendo para facilitar la tarea del cirujano en lo referente a la hemostasia quirúrgica y la prevención de la lesión recurrencial. Así, el uso de dispositivos como el bisturí ultrasónico (Ultracision ${ }^{\mathrm{TM}} \mathrm{CS}-14 \mathrm{C}$, Ethicon Endo-Surgery, USA) o el sistema de sellado bipolar (Ligasure ${ }^{\mathrm{R}}$ Valleylab, CO, USA), como la aparición de los sistemas de monitorización intraoperatoria electrofisiológica del nervio recurrente $\left(\mathrm{NIM}^{\mathrm{R}}\right.$ Medtronic, USA), es hoy en día habitual en la mayoría de las Unidades dedicadas a la cirugía endocrina ${ }^{3-5}$.

El objetivo de nuestro trabajo es estudiar el impacto de estas tecnologías en cirugía tiroidea tras su introducción en nuestra Unidad, una vez aprobada por la Comisión de Evaluación de Nuevas Tecnologías, tanto desde el punto de vista de su eficiencia así como de los resultados clínicos.

\section{Material y Método}

Se realiza un estudio retrospectivo y comparativo de una muestra de tres grupos de pacientes sometidos a tiroidectomía total con diagnóstico preoperatorio de bocio multinodular o hipertiroidismo, correspondientes a tres períodos de tiempo. Se incluyeron en el estudio aquellos pacientes con patología tiroidea benigna susceptible de tiroidectomía total (bocio multinodular, bocio multinodular tóxico y enfermedad de Graves). Se excluyeron los pacientes sometidos a hemitiroidectomía, aquellos con diagnóstico preoperatorio de malignidad, bocio recidivado, así como los casos en que hubo que realizar otro procedimiento asociado (linfadenectomía, paratiroidectomía, exéresis de quiste tirogloso). Los datos de los pacientes fueron obtenidos a partir de la historia clínica electrónica así como del Conjunto Mínimo Básico de Datos. En ningún caso se ha hecho uso de datos personales o identificativos de los pacientes. Los autores declaran que se han seguido los protocolos establecidos por el hospital para acceder a los datos de las historias clínicas para poder realizar este tipo de publicación con finalidad de investigación. El estudio ha recibido el aval del Comité Ético de Investigaciones Clínicas del Hospital Morales Meseguer.

Los grupos fueron:

1. Grupo de 96 pacientes sometidos a tiroidectomía total en el período 2004-2007. Los pacientes fueron remitidos a cirugía desde la consulta de endocrinología tras estudio clínico, analítico, radiológico y, en su caso, citológico. Una vez establecida la indicación de cirugía los pacientes fueron intervenidos, realizándose tiroidectomía total o casi total reglada. En todos los pacientes se realizó estudio anestésico preoperatorio así como valoración laringoscópica mediante laringoscopia indirecta pre y postoperatoria. Respecto al control postoperatorio, se realizó una laringoscopia al mes de la intervención y, en los casos de parálisis/paresia, un control bimensual hasta la resolución o hasta el año. Durante este período, se utilizaron técnicas hemostáticas basadas en electrocoagulación, ligaduras y hemoclips.

2. Ciento ocho pacientes intervenidos en el período 
2008-2010. Los pacientes fueron intervenidos según los mismos criterios y con las mismas premisas del período anterior. En este período la hemostasia quirúrgica se realizó fundamentalmente con el sistema de disección-coagulación ultrasónica o bisturí armónico (Harmonic Ultracision ${ }^{\mathrm{R}}$ ).

3. Grupo de 82 pacientes correspondientes al período 2011-2012. Durante este período se utilizó el mismo instrumento para hemostasia del período anterior. Además, se realizó monitorización intraoperatoria del nervio laríngeo recurrente con el sistema $\mathrm{NIM}^{\mathrm{R}}$. El procedimiento consistió en identificación y electroestimulación de los nervios vagos y laríngeos recurrentes a una intensidad de 1-2 mA, antes y después de la exéresis glandular. Se registraron los valores de latencia y amplitud de onda en ambos momentos.

Todos los pacientes intervenidos fueron informados del procedimiento y firmaron el correspondiente consentimiento informado.

Tras el alta hospitalaria, los pacientes fueron seguidos en las consultas de Cirugía General y Endocrinología durante un período de 1 año, tras el cual fueron remitidos a su médico de familia (casos benignos). Los casos en los que el estudio anatomopatológico de la pieza evidenció la presencia de una neoplasia fueron remitidos a Medicina Nuclear para tratamiento con $\mathrm{I}^{131} \mathrm{y}$ seguidos posteriormente por Endocrinología y Cirugía. Durante este período se dosificó el tratamiento con tiroxina y se evaluaron y siguieron las complicaciones (hipoparatiroidismo y lesión recurrencial).

Analizamos los tres períodos y realizamos un estudio comparativo respecto a las siguientes variables: sexo, edad, diagnóstico, tipo de cirugía, diagnóstico anatomopatológico, complicaciones, estancia postoperatoria, tiempo quirúrgico empleado y coste total por paciente. Respecto a las complicaciones, definimos hipocalcemia cuando la cifra de calcio iónico fue inferior a $4 \mathrm{mg} / \mathrm{dl}(1 \mathrm{mmol} / \mathrm{lt})$, medido a las $24 \mathrm{~h}$ de la intervención quirúrgica. Se consideró hipoparatiroidismo definitivo la hipocalcemia mantenida 6 meses tras la intervención. Se registró asimismo la presencia de edema, seroma o hematoma de la herida quirúrgica independientemente de la necesidad de reoperación. Referente a la parálisis recurrencial, ésta se consideró definitiva si persistía al año de la cirugía. Los costes se obtuvieron a partir de los datos de contabilidad analítica suministrados por el departamento de Control de Gestión del hospital. Según esto, los costes por paciente se desglosan en: material, laboratorio, hospitalización, personal, servicios intermedios y quirófano, correspondiendo a este último un coste de 4,028 euros/min.

\section{Análisis estadístico}

Se realizó un muestreo sistemático, con sustitución, en su caso, por el inmediatamente anterior. Las variables se expresan como medias \pm desviación estándar, frecuencias absolutas y relativas. La comparación entre variable cualitativa y cuantitativa de 3 opciones, se ha realizado previa comprobación de normalidad de las variables cuantitativas, aplicándose la prueba ANOVA de un factor o Kruskal Wallis. La comparación entre variables cualitativas se ha realizado mediante la prueba $\chi^{2}$ de tendencia lineal. Los análisis se han realizado con el paquete estadístico SPSS versión 15.0 para Windows.

\section{Resultados}

Se obtuvo una muestra de 96 pacientes en el primer período (2004-2007). La distribución por sexos fue de 90 mujeres $(93,8 \%)$ y 6 hombres $(6,3 \%)$ y la edad media de los pacientes de $52,4 \pm$ 13,1 años. Del segundo período (2008-2010) obtuvimos una muestra de 108 pacientes, distribuidos en 90 mujeres $(83,3 \%)$ y 18 hombres $(16,7 \%)$. La edad media en este período fue de 47,7 $\pm 13,7$ años. En el tercer período (2011-2012) obtuvimos una muestra de 82 pacientes, 68 mujeres $(82,9 \%)$ y 14 hombres $(17,1 \%)$ con una edad media de 48,4 $\pm 14,1$ años.

Los tres grupos de pacientes fueron homogéneos en cuanto a diagnóstico clínico y anatomopatológico, tipo de cirugía y cirujano responsable. En todos los casos, la indicación quirúrgica más frecuente fue el bocio multinodular (BMN). Se realizó cirugía primaria en la mayoría de casos y el mayor porcentaje de pacientes fue intervenido por un cirujano staff perteneciente a la unidad de cirugía endocrina. Respecto al estudio anatomopatológico de la pieza quirúrgica, decir que no hubo diferencias significativas entre los tres grupos en el número de cánceres identificados (Tabla 1).

Respecto a la evaluación de las complicaciones, hemos analizado las tasas de hemorragia, hipocalcemia y parálisis recurrencial y observamos que hay una disminución en el número de parálisis recurrenciales en el tercer período respecto a los otros dos, aunque la diferencia no alcanza significación estadística $(4,9 \%$, en comparación a 12,5 y $11,1 \%$ en el primer y segundo período respectivamente). De la misma manera, tanto en la forma transitoria como permanente, en el segundo y tercer período registramos menos casos de hipocalcemia (Tabla 2). La Tabla 3 nos refleja las diferencias obtenidas entre los tres períodos. Respecto a la estancia postoperatoria, observamos una disminución significativa en el segundo y tercer período respecto al primero. 
Tabla 1. Distribución homogénea entre los tres grupos de pacientes de las principales variables demográficas y clínicas

\begin{tabular}{|c|c|c|c|c|}
\hline & $\begin{array}{c}\text { 2004-7 } \\
\text { n: } 96\end{array}$ & $\begin{array}{c}2008-10 \\
n: 108\end{array}$ & $\begin{array}{c}\text { 2011-12 } \\
n: 82\end{array}$ & p \\
\hline $\begin{array}{l}\text { Sexo } \\
\text { Mujer } \\
\text { Hombre }\end{array}$ & $\begin{array}{l}90(93 \%) \\
6\end{array}$ & $\begin{array}{l}90(83 \%) \\
18\end{array}$ & $\begin{array}{l}68(82 \%) \\
14\end{array}$ & 0,124 \\
\hline Edad & $52,4 \pm 13$ & $47,7 \pm 13$ & $48,4 \pm 14$ & 0,196 \\
\hline $\begin{array}{l}\text { Diagnóstico } \\
\text { BMN } \\
\text { BMN tóxico } \\
\text { Graves }\end{array}$ & $\begin{array}{l}82(85 \%) \\
14 \\
0\end{array}$ & $\begin{array}{l}74(68 \%) \\
20 \\
14\end{array}$ & $\begin{array}{l}64(78 \%) \\
10 \\
8\end{array}$ & 0,154 \\
\hline $\begin{array}{l}\text { Tipo de cirugía } \\
\text { Primaria } \\
\text { Cirugía de recidiva }\end{array}$ & $\begin{array}{l}94(97 \%) \\
2\end{array}$ & $\begin{array}{l}106(98,1) \\
2\end{array}$ & $\begin{array}{l}80(97 \%) \\
2\end{array}$ & 0,914 \\
\hline $\begin{array}{l}\text { Cirujano } \\
\text { Staff unidad } \\
\text { Staff no unidad } \\
\text { Residente }\end{array}$ & $\begin{array}{l}82(85 \%) \\
14 \\
0\end{array}$ & $\begin{array}{l}92(85 \%) \\
14 \\
2\end{array}$ & $\begin{array}{l}74(90 \%) \\
2 \\
6\end{array}$ & 0,785 \\
\hline $\begin{array}{l}\text { Anatomia patológica } \\
\text { Bocio nodular } \\
\text { Bocio difuso } \\
\text { Microcarcinoma papilar } \\
\text { Carcinoma papilar } \\
\text { Carcinoma folicular } \\
\text { Otros }\end{array}$ & $\begin{array}{l}84(87 \%) \\
0 \\
8 \\
4 \\
0 \\
0\end{array}$ & $\begin{array}{r}70(64 \%) \\
12 \\
8 \\
12 \\
0 \\
6\end{array}$ & $\begin{array}{l}48(58 \%) \\
8 \\
16 \\
0 \\
6 \\
4\end{array}$ & 0,868 \\
\hline
\end{tabular}

Tabla 2. Análisis de las complicaciones en los tres períodos

\begin{tabular}{|lcccc|}
\hline & $\begin{array}{c}\mathbf{2 0 0 4 - 7} \\
\mathbf{n : ~ 9 6}\end{array}$ & $\begin{array}{c}\mathbf{2 0 0 8 - 1 0} \\
\mathbf{n : ~ 1 0 8}\end{array}$ & $\begin{array}{c}\mathbf{2 0 1 1 - 1 2} \\
\mathbf{n : ~ 8 2}\end{array}$ & $\mathbf{p}$ \\
Hemorragia/hematoma & & & & \\
Sí & 4 & 2 & 4 & 0,888 \\
No & $92(95 \%)$ & $106(98,1)$ & $78(95 \%)$ & \\
Hipocalcemia & $40(41 \%)$ & $30(27 \%)$ & $20(24 \%)$ & 0,076 \\
$\quad$ Transitoria & 30 & 26 & 16 & \\
Permanente & 10 & 4 & 4 & \\
Parálisis recurrencial & & & 3 & \\
$\quad$ Unilateral transitoria & 6 & 8 & 1 & \\
Unilateral definitiva & 4 & 2 & 0 & \\
Bilateral transitoria & 2 & 0 & 0 & \\
Bilateral definitiva & 0 & $12(11 \%)$ & $4(4,9 \%)$ & \\
Total & $12(13 \%)$ & & 3 & \\
\hline
\end{tabular}

Tabla 3. Las diferencias entre los tres períodos referentes a estancia (días), tiempo quirúrgico (minutos) y costes (euros) alcanzan significación estadística

\begin{tabular}{|lcccc|}
\hline & $\mathbf{2 0 0 4 - 7}$ & $\mathbf{2 0 0 8 - 1 0}$ & $\mathbf{2 0 1 1 - 1 2}$ & p \\
& $\mathbf{n : 9 6}$ & $\mathbf{n : ~ 1 0 8}$ & $\mathbf{n : ~ 8 2}$ & $<0,001$ \\
Estancia postoperatoria & $3,9 \pm 1$ & $2,5 \pm 1$ & $2,7 \pm 2$ & $<0,001$ \\
Tiempo quirúrgico & $101,9 \pm 22$ & $70,1 \pm 24$ & $70,8 \pm 29$ & $<0,001$ \\
\hline Costes totales & $2.555 \pm 714$ & $2.919 \pm 674$ & $3.216 \pm 772$ & $<$ \\
\hline
\end{tabular}


De la misma manera, cuando analizamos el tiempo quirúrgico vemos una reducción aproximada de unos 30 min por paciente en el segundo y tercer período respecto al primero. Por último, observamos también diferencias significativas en el coste total por paciente. Así, en el segundo período tenemos un incremento desde $2.555 \pm 714$ euros hasta $2.919 \pm$ 674 . En el tercer período vuelve a aumentar el coste por paciente hasta $3.216 \pm 772$ euros.

\section{Discusión}

Billroth, Kocher, Halsted y otros autores representan el inicio de la era moderna en cirugía tiroidea. Hablamos de mediados del siglo XIX y, desde entonces, las principales preocupaciones del cirujano endocrino no han variado en lo que respecta a la cirugía de la glándula tiroides: hemorragia, hipoparatiroidismo y lesión de los nervios laríngeos ${ }^{1}$. Una cuidadosa técnica quirúrgica, asegurando la hemostasia mediante ligaduras y hemoclips ayudados de instrumentos eléctricos convencionales, han sido tradicionalmente, la base para conseguir una correcta hemostasia. En lo que respecta a la indemnidad de las glándulas paratiroides y los nervios laríngeos, la identificación sistemática de estas estructuras y su cuidadoso manejo son la principal aportación que puede hacer el cirujano. Con todo, tasas de hasta un 4\% para problemas hemorrágicos y hasta un 30\% para hipoparatiroidismo transitorio siguen publicándose en la literatura. Respecto a la lesión nerviosa en patología benigna, cifras de hasta un $3 \%$ para parálisis definitiva siguen comunicándose $\mathrm{e}^{2,3}$.

En los últimos años, han ido apareciendo diversos instrumentos con objeto de ayudar al cirujano a mejorar sus resultados en cirugía tiroidea. Dicha tecnología se va imponiendo en la mayoría de las unidades dedicadas al tratamiento de esta patología, haciendo necesaria la aparición de estudios que evalúen la eficacia y eficiencia de la misma (costebeneficio $)^{6,7}$. Así, en lo referente a la hemostasia, el bisturí ultrasónico y el sistema de sellado de vasos bipolar son utilizados en la actualidad por la mayoría de las unidades de cirugía endocrina ${ }^{8-15}$. En nuestra Unidad, comenzamos a utilizar en el año 2007 el bisturí ultrasónico. El mecanismo de acción de este dispositivo se basa en una transferencia de energía a los tejidos por vibración (55.000 veces/seg). Ello provoca una desnaturalización de proteínas por rotura de puentes de hidrógeno a temperaturas de $60-80^{\circ} \mathrm{C}$ produciéndose el coágulo ${ }^{5}$.

Por otro lado, respecto a la prevención de las lesiones del nervio laríngeo recurrente, la atención se ha dirigido hacia el uso de instrumentos de monitorización intraoperatoria. Su objetivo es ayudar a preservar la función del nervio mediante su identificación electrofisiológica sobre todo en casos de grandes bocios, reintervenciones o cirugía de cáncer ${ }^{16-20}$. Nuestro estudio trata de evaluar el impacto de estas nuevas tecnologías en cirugía tiroidea. Analicemos pues, por separado, dicho impacto:

\section{Uso de bisturí ultrasónico}

Los datos publicados indican que el uso del bisturí ultrasónico implica un menor tiempo quirúrgico $^{8-15}$, siendo controvertidos los datos referentes a coste y tasa de complicaciones. Así, mientras algunos grupos hablan de una menor tasa de complicaciones ${ }^{9,12,15,21}$, otros dicen que no hay diferencias significativas ${ }^{8,10,11,13,14}$. En nuestro estudio vemos que al comparar los dos primeros grupos de pacientes, observamos un significativo ahorro de tiempo quirúrgico en el segundo grupo a expensas de un aumento del coste por paciente. Podemos hablar de un aumento de gasto de 39.312 euros para el total del grupo de pacientes y una ganancia global de 55,8 h. No hubo diferencias significativas en cuanto a las complicaciones aunque sí queremos resaltar una tendencia a la disminución de los hipoparatiroidismos $(41,7 \%$ vs $27,8 \%)$. Un dato llamativo en nuestro estudio es que la estancia media de los pacientes en el segundo grupo fue significativamente menor que en el primero (2,5 $\pm 1,2$ días vs $3,9 \pm 1,9$ días). Atribuir esta diferencia al uso del bisturí ultrasónico, aunque coincidiría con resultados de otros autores ${ }^{21}$, nos parece precipitado antes de realizar un estudio aleatorizado donde puedan controlarse otras variables.

\section{Neuromonitorización intraoperatoria}

La parálisis del nervio laríngeo recurrente, en su forma permanente, es una infrecuente pero grave complicación de la cirugía tiroidea. Las cifras oscilan entre 0,2 y más del 3\% de lesión permanente según el tipo de enfermedad tiroidea, que se trate de cirugía primaria o reintervención, o bien, se realice una tiroidectomía total o subtotal ${ }^{2-5}$. La identificación sistemática y una cuidadosa disección nerviosa continúan siendo los principales factores de prevención de esta complicación. Han pasado más de 30 años desde que los primeros grupos empezaron a utilizar la monitorización electrofisiológica del nervio recurrente durante la intervención quirúrgica, con resultados dispares en cuanto a prevención de lesiones ${ }^{2-5,16-20}$. En los últimos años, vuelve a estar en auge el uso de estos dispositivos. Su uso se basa principalmente en facilitar la identificación del nervio, ayudar en su disección y, lo más importante, pronosticar su función tras la intervención quirúrgica $^{3-5}$. Respecto a esto último, los autores coinciden en su alto valor predictivo negativo, es 
decir, su capacidad para predecir función nerviosa postoperatoria normal $(97-99 \%)^{2,16-18}$. En nuestro tercer grupo de pacientes utilizamos tanto el bisturí ultrasónico como la neuromonitorización intraoperatoria. En lo referente a estancia y tiempos quirúrgicos los datos no difieren significativamente de los del segundo grupo y sí hay un aumento de gasto de 661 euros/paciente respecto al primer grupo y 297 euros/paciente respecto al segundo. Tal como describíamos en nuestros resultados, tenemos menos parálisis recurrenciales en el grupo de pacientes con neuromonitorización. Probablemente el tamaño muestral sea responsable de la ausencia de diferencias significativas.

Vemos pues, en nuestros resultados, el impacto que ha supuesto el uso en nuestra Unidad de estas nuevas tecnologías. Es evidente que el coste por paciente aumenta. Sin embargo, el considerable ahorro de tiempo quirúrgico que supone el uso del bisturí ultrasónico es un dato a tener en cuenta por lo que supone en el aumento de la disponibilidad de un recurso tan escaso como es el quirófano. Por otro lado, la neuromonitorización intraoperatoria supone una ayuda adicional a la técnica quirúrgica. Nosotros hemos observado menos lesiones recurrenciales aunque, como ya apuntan otros autores, hay que recordar que todavía no se han publicado diferencias significativas respecto a la prevención de lesión nerviosa en comparación con una cuidadosa técnica quirúrgica ${ }^{20}$. Una tendencia hacia la disminución de las complicaciones que estamos tratando con el uso de estas nuevas tecnologías, aún sin significación estadística, ha sido comunicada recientemente ${ }^{22}$. Futuros estudios controlados nos confirmarán si las diferencias son significativas.

\section{Referencias}

1. Alam Hannan S. The magnificent seven: a history of modern thyroid surgery. Int J Surg. 2006;4:187-91.

2. Dralle H, Sekulla C, Lorenz K, Brauckhoff M, Machens A. German IONM Study Group. Intraoperative monitoring of the recurrent laryngeal nerve in thyroid surgery. World J Surg. 2008;32:1358-66.

3. Dionigi G, Bacuzzi A, Bertocchi V, Carrafiello G, Boni L, Rovera F, et al. Safe incorporation of new technologies in thyroid surgery. Expert Rev Med Devices 2008;5:747-58.

4. Dionigi G, Bacuzzi A, Boni L, Rovera F, Piantanida E, Tanda ML, et al. Influence of new technologies on thyroid surgery: state of the art. Expert Rev Med Devices 2005;2:547-57.

5. Becker AM, Gourin CG. New technologies in thyroid surgery. Surg Oncol Clin North Am. 2008;17:233-48.

6. Gómez-Palacios A, Barrios B, Gutiérrez MT, Expósito
A, Gómez-Zabala J, Roca B, y cols. Morbilidad y costes en las tiroidectomías totales. Mejora del valor del proceso mediante el cambio de gestión. Rev Calid Asist. 2012;27:161-8.

7. Pardal-Refoyo JL. Comentarios sobre morbilidad y costes en tiroidectomía total. Rev Calid Asist. 2012;27:3634.

8. Cirocchi R, Boselli C, Guarino S, Sanguinetti A, Trastulli S, Desiderio J, et al. Total thyroidectomy with ultrasonic dissector for cancer: multicentric experience. World J Surg Oncol. 2012;10:1-5.

9. Sista F, Schietroma M, Ruscitti C, De Santis G, De Vita F, Carlei F, et al. New ultrasonic disector versus conventional hemostasis in thyroid surgery: a randomized prospective study. Adv Surg Tech. A 2012;22:2204.

10. Gentileschi P, D’Ugo S, Iaculli E, Gaspari AL. Harmonic Focus ${ }^{\mathrm{TM}}$ versus "knot tying" during total thyroidectomy: a randomized trial. Updates Surg 2011;63:27781.

11. Cirocchi R, D'Ajello F, Trastulli S, Santoro A, Di Rocco G, Vendettuoli D, et al. Meta-analysis of thyroidectomy with ultrasonic dissector versus conventional clamp and tie. World J Surg Oncol. 2010;8:112.

12. Mourad M, Rulli F, Robert A, Scholtes JL, De Meyer M, De Pauw L. Randomized clinical trial on Harmonic Focus shears versus clamp-and-tie technique for total thyroidectomy. Am J Surg. 2011;202:168-74.

13. Sebag F, Fortanier C, Ippolito G, Lagier A, Auquier $\mathrm{P}$, Henry JF. Harmonic scalpel in multinodular goiter surgery: impact on surgery and costs analysis. J Laparoendosc Adv Surg Tech. A 2009;19:171-4.

14. Ecker T, Carvalho AL, Choe JH, Walosek G, Preuss KJ. Hemostasis in thyroid surgery: Harmonic scalpel versus other techniques-a meta-analysis. Otolaryngol Head Neck Surg. 2010;143:17-25.

15. Ferri E, Armato E, Spinato G, Spinato R. Focus Harmonic scalpel compared to conventional haemostasis in open total thyroidectomy: A prospective randomized trial. Int J Otolaryngol. 2011;1-7.

16. Hermann M, Hellebart C, Freissmuth M. Neuromonitoring in thyroid surgery. Prospective evaluation of intraoperative electrophysiological responses for the prediction of recurrent laryngeal nerve injury. Ann Surg. 2004;240:9-17.

17. Sturgeon C, Sturgeon T, Angelos P. Neuromonitoring in thyroid surgery: Attitudes, usage patterns and predictors of use among endocrine surgeons. World J Surg. 2009;33:417-25.

18. Goretzki PE, Schwarz K, Brinkmann J, Wirowski D, Lammers BJ. The impact of intraoperative neuromonitoring (IONM) on surgical strategy in bilateral thyroid diseases: is it worth the effort? World J Surg. 2010;34:1274-84.

19. Dionigi G, Bacuzzi A, Boni L, Rausei S, Rovera F, Dionigi R. Visualization versus neuromonitoring of 
B. FLORES P. y cols.

recurrent laryngeal nerves during thyroidectomy: what about the costs? World J Surg. 2012;36:748-54.

20. Angelos P. Ethical and medicolegal issues in neuromonitoring during thyroid and parathyroid surgery: a review of the recent literature. Curr Opin. 2012;24:1621.
21. Pardal-Refoyo JL. Sistemas de hemostasia en cirugía tiroidea y complicaciones. Acta Otorrinolaringol Esp. 2011;62:339-46.

22. Pardal-Refoyo JL. Hemostasis and neuromonitoring as patient safety measures in thyroid surgery. Rev Calid Asist. 2013. Doi:10.1016/j.cali.2012.10.008. 